\title{
Níveis de lisina com dois balanços eletrolíticos para frangos de corte na fase de crescimento ( 22 a 40 dias)
}

\section{Evandro Campestrini ${ }^{1}$, Maria José Baptista Barbosa ${ }^{2}$, Ricardo Vianna Nunes ${ }^{3}$, Luís Daniel Giusti Bruno ${ }^{2}$, Wagner Thiago Mozer da Silva ${ }^{4}$, Matias Djalma Appelt ${ }^{4}$}

\author{
${ }^{1}$ Pós-graduação em Zootecnia - UEM. \\ 2 Departamento de Zootecnia - UEM. \\ ${ }^{3}$ Departamento de Zootecnia - UNIOESTE. \\ 4 Curso de Graduação em Zootecnia - UNIOESTE.
}

RESUMO - Objetivou-se avaliar os níveis de lisina para frangos de corte na fase de crescimento (22 a 40 dias de idade) utilizando-se dois balanços eletrolíticos. Foram utilizados 640 frangos de corte machos de 21 dias de idade, linhagem Cobb, distribuídos em delineamento inteiramente casualizado, em esquema fatorial $5 \times 2$, composto de cinco níveis de lisina (1,062, 20, 40 e $60 \%$ acima e $20 \%$ abaixo do recomendado) e dois balanços eletrolíticos (190 e $255 \mathrm{mEq} / \mathrm{kg}$ ), com quatro repetições e 16 aves por unidade experimental. Não foi observada interação das variáveis estudadas, porém, os níveis de lisina influenciaram o peso final, o ganho de peso e a conversão alimentar e afetaram de forma linear o consumo de ração. Os melhores ajustes foram observados quando se utilizou o modelo descontinuo LRP, no qual valores acima de 1,089 e 1,14\% de lisina não melhoraram o ganho de peso e tampouco a conversão alimentar. Entre os parâmetros sangüíneos avaliados, apenas o ácido úrico foi influenciado pelos níveis de lisina. Os rendimentos de carcaça e de cortes nobres não diferiram significativamente entre os níveis de lisina e os balanços eletrolíticos, contudo, a gordura abdominal foi influenciada pelo balanço eletrolítico da dieta. Níveis acima de 1,089 e 1,14\% de lisina, respectivamente, não melhoram o desempenho das aves. Os níveis de lisina testados não influenciaram o rendimento de carcaça nem de cortes nobres.

Palavra-chave: aminoácidos, aves de corte, balanço eletrolítico, lisina

\section{Lysine level with two electrolytes balance for broiler chickens in the growing phase (22 a 40 day)}

\begin{abstract}
The lysine levels for chickens in the growing phase (22 to 40 days of age) by using two different electrolytes balance were evaluated. Sixty-four hundred Cobb broiler male of 21 days old were allotted to a completely randomized design as a factorial arrangement of treatments, five lysine levels $(1.062,20,40$, and $60 \%$ above and $20 \%$ below of recommended levels) and two electrolytes balance (190 and $255 \mathrm{mEq} / \mathrm{kg}$ ), with four replicates and 16 broilers for experimental unit. There was no interaction among the variables studied, however the lysine levels affected final weight, weight gain and the feed:gain ratio and feed intake, however, the best adjustments were observed when the discontinue LRP model was used, where values above 1.089 and $1.14 \%$ of lysine did not increase weight gain and feed:gain ratio. Concerning the blood parameters, only uric acid was affected by lysine levels, but no difference was observed for carcass and prime cuts yield, the abdominal fat was influenced by the dietary electrolyte balance. Lysine levels above 1.089 and 1.14 for weight gain and feed:gain ratio, respectively, did not impair broiler performance. The lysine levels affected neither carcass yield and nor prime cuts yield.
\end{abstract}

Key Words: amino acids, cut birds, electrolytes balance, lysine

\section{Introdução}

O objetivo na produção de frangos de corte tem se modificado ao longo dos últimos anos, com o aumento do consumo interno e das exportações de partes desossadas em detrimento da carcaça inteira. Dessa forma, o rendimento de cortes como peito e pernas, aliado a carcaças com menor quantidade de gordura, passou a ser fundamental para a indústria.

A algum tempo atrás, as dietas das aves eram formuladas com base em proteína bruta, o que poderia resultar em grandes perdas, tanto energéticas quanto econômicas, principalmente quando ocorriam desbalanceamento entre aminoácidos. Atualmente, recomenda-se formular dietas 
com aminoácidos na proporção ideal, de modo que não existam deficiências ou excessos. Vários estudos têm sido realizados com o objetivo de determinar as exigências de vários aminoácidos principalmente lisina, metionina, treonina, triptofano e arginina.

Tem sido observado que a utilização de níveis mais altos de lisina nas dietas de crescimento e terminação aumentam a quantidade de carne de peito (Mendes, 1990). Segundo as recomendações do NRC (1994), a exigência de lisina na fase inicial é de $1,10 \%$, reduzindo-se para $1,00 \%$ na fase de crescimento ( $22-42$ dias) e de $0,85 \%$ na fase final ( 42 49 dias), diminuição esta que parece muito acentuada e que vários autores têm questionado.

Com o objetivo de verificar esses níveis, Almeida et al. (2002) não observaram diferença para ganho de peso, conversão alimentar e rendimento de carcaça quando utilizaram níveis de lisina acima do recomendado pelo NRC (1994) (100, 110 e $120 \%$ para as rações inicial, crescimento e terminação, respectivamente), porém observaram que os níveis mais altos de lisina resultaram em menores porcentagens de gordura abdominal quando comparados aos níveis mais baixos, nas fêmeas.

Diversos estudos têm demonstrado a importância da arginina para o crescimento, sistema imune, sistema locomotor, formação das penas (Corzo \& Kidd, 2003; Gadelha, 2004; Kidd, 2005) e indicando também que o desbalanço entre os aminoácidos arginina e lisina são prejudiciais às aves, devido ao antagonismo existente entre esses aminoácidos. Alguns autores demonstraram que a exigência de arginina é aumentada pelo excesso de lisina na dieta.

Quando os níveis de lisina são excessivamente altos, reduzindo a relação entre arginina e lisina, ocorre excreção renal de arginina (Nesheim, 1968; Borman, 1971), aumento da concentração plasmática e tecidual de lisina (como proteína), diminuição da concentração de potássio intracelular e aumento do nível de cloro plasmático (Calvert \& Austic, 1981).

A temperatura ambiental influencia o consumo de alimento dos frangos de corte e o balanço eletrolítico dos mesmos, podendo interagir com a resposta aos níveis dietéticos de arginina e lisina. Brake et al. (1998) trabalhando com frangos de corte de 20 a 41 dias de idade, observaram que em ambientes quentes houve melhoria da conversão alimentar com o aumento da relação arginina e lisina, mantendo-se constante a lisina, enquanto que em ambientes frios, a maior relação arginina e lisina melhorou o ganho de peso. Por outro lado, o aumento da concentração de cloreto de sódio na ração reduziu a necessidade de aumento na relação arginina e lisina para obtenção de melhor conversão alimentar.
É bem conhecido que o balanço eletrolítico influencia o crescimento dos frangos de corte. As proporções dietéticas de sódio, potássio e cloro são importantes determinadores do equilíbrio ácido-básico. O sódio e potássio são alcalinogênicos e o cloro, acidogênico.

Barros et al. (2004), com o objetivo de determinar as exigências de sódio para frangos de corte machos e fêmeas, nos períodos de 22 a 42 e de 43 a 53 dias de idade, utilizando uma dieta basal (3.100 e $3.210 \mathrm{kcal} \mathrm{EM} / \mathrm{kg}$ e 19,6 e 18,6\% de PB) deficiente em sódio (0,017\%), suplementada com $\mathrm{NaCl}$, correspondente aos níveis 0,$077 ; 0,137 ; 0,197 ; 0,257$ e $0,317 \%$ de sódio total, observaram que em ambos os sexos, os níveis de sódio influenciaram o ganho de peso, a conversão alimentar e a umidade da cama.

Veldkamp et al. (1999) estudando os efeitos da temperatura ambiental $\left(15^{\circ} \mathrm{Ce} 30^{\circ} \mathrm{C}\right)$, relação arginina e lisina $(1,00$ e 1,25 ) e o balanço eletrolítico (164 e $254 \mathrm{mEq} / \mathrm{kg}$ ) em perus machos, observaram que, a temperatura influenciou negativamente o consumo de ração e o ganho de peso, com o aumento da relação arginina lisina, aumentou o consumo de ração e consequentemente o ganho de peso, porém, o balanço eletrolítico não afetou os parâmetros de desempenho, mas teve interação significativa com a temperatura. Os autores concluíram que o desempenho das aves foi comprometido pela alta temperatura, e que alterações na relação arginina lisina e no balanço eletrolítico da dieta não aliviaram o prejuízo causado ao desempenho das aves. Os níveis dietéticos de arginina parecem ser importantes quando o requerimento de lisina dietética é relativamente marginal.

Objetivou-se com este trabalho determinar o nível de lisina utilizando-se dois balanços eletrolíticos para frangos de corte na fase de crescimento e observar a influência desses fatores sobre os parâmetros sanguíneos, rendimento de carcaça e cortes nobres.

\section{Material e Métodos}

O experimento foi conduzido no setor de Avicultura da Fazenda Experimental da Linha Guará, do Centro de Ciências Agrárias da Universidade Estadual do Oeste do Paraná - UNIOESTE, localizada em Marechal Cândido Rondon, Paraná, no período de 9 a 28 de outubro de 2005. Foram utilizados 640 pintos de corte da linhagem Cobb com 22 dias de idade, distribuídos em delineamento inteiramente casualizado, em esquema fatorial $5 \times 2$, composto de cinco níveis de lisina e dois níveis de balanço eletrolítico, com quatro repetições por tratamento e 20 aves por unidade experimental.

As aves foram criadas em 40 boxes $(1,3 \mathrm{~m} \times 1,2 \mathrm{~m}) \mathrm{em}$ um galpão convencional de $20 \mathrm{~m} \times 8 \mathrm{~m}$, com pé-direito de $3 \mathrm{~m}$, 
disposto no sentido leste-oeste, coberto com telha de barro do tipo francesa, com lanternin, piso de concreto, paredes laterais de alvenaria com $0,30 \mathrm{~m}$ de altura completadas com tela de arame até o telhado e paredes das extremidades fechadas até o teto (telhado) com alvenaria. Foram alojadas 16 aves por boxe, em densidade de 10,26 aves $/ \mathrm{m}^{2}$, utilizando-se como cama maravalha de madeira reutilizada uma vez.

A temperatura e a umidade relativa do ar durante o período experimental foram medidas por termômetro de máxima e mínima instalados no interior do galpão (Tabela 1).

No primeiro dia de idade, as aves foram vacinadas no incubatório contra a doença de Marek, bouba e bronquite infecciosa e, aos 7 e 14 dias de idade, foram vacinadas via água, no galpão, contra a doença de gumboro (Lohmann Animal Health International).

No período de 1 a 21 dias de idade, as aves foram alojadas em um aviário de alvenaria, onde receberam água e ração inicial à vontade, de acordo com a exigência de Rostagno et al. (2005). As rações experimentais foram formuladas a base de milho, farelo de soja e glúten de milho e suplementadas com aminoácidos sintéticos de modo a apresentar níveis de lisina digestível de 1,062, 20,40 e 60\% acima e $20 \%$ abaixo desse valor.

Aos 21 dias de idade, as aves foram pesadas e distribuídas aleatoriamente nas unidades experimentais. O peso inicial médio das aves foi de $873,75 \pm 11,49 \mathrm{~g}$.

Do $21^{\circ}$ ao $40^{\circ}$ dia de idade, foram avaliados o peso final (PF), o ganho de peso (GP), o consumo de ração (CR) e a conversão alimentar (CA). Aos 40 dias de idade, realizou-se coleta de sangue para análise de proteína total, ácido úrico e cálcio utilizando-se duas aves por boxe, totalizando 80 amostras. Para padronização da coleta de sangue, adotou-se metodologia utilizada por Conhalato et al., (2000): retirada da ração pela manhã por 1 hora; retomada da alimentação por 1 hora; novamente, retirada da ração por 1 hora; retomada da alimentação por mais 1 hora; jejum de 3 horas; e finalmente coleta do sangue via punção braquial.

Depois de coletado, o sangue foi armazenado em tubos de ensaio e encaminhado ao laboratório para centrifugação a $2.000 \mathrm{rpm}$ por 15 minutos para retirada do soro sanguíneo por meio de micropipeta. O soro foi armazenado em tubos para posterior análise dos teores de proteína total, ácido úrico e cálcio utilizando-se kit comercial. Regularmente, registraram-se o peso e o número de aves mortas e a quantidade de sobras de ração do boxe em que a ave estava alojada para ajuste do consumo de ração e da conversão alimentar.

A avaliação de rendimento de carcaça foi realizada utilizando-se duas aves aos 40 dias de idade, retiradas ao acaso de cada unidade experimental, totalizando 80 aves,
Tabela 1 - Temperatura e umidade relativa (UR) do ar no interior do galpão durante o período experimental

\begin{tabular}{lccc}
\hline & \multicolumn{3}{c}{ Idade das aves (dias) } \\
\cline { 2 - 4 } Temperatura do ar $\left({ }^{\circ} \mathrm{C}\right)$ & 22 a 28 & 29 a 35 & 36 a 40 \\
\hline Máxima média & 30,07 & 26,37 & 28,53 \\
Mínima média & 23,62 & 20,94 & 21,71 \\
UR máxima (\%) & 78,96 & 87,93 & 87,85 \\
UR mínima (\%) & 49,96 & 64,07 & 65,35 \\
\hline
\end{tabular}

que foram abatidas após jejum de 6 horas. As aves foram pesadas e marcadas individualmente, sacrificadas, sangradas pela veia jugular, depenadas e evisceradas. As carcaças, sem pés, cabeça e pescoço, foram pesadas e separadas nos cortes peito, coxa e sobrecoxa. O fígado, os rins e a gordura abdominal depositada em volta da cloaca foram retirados e pesados. Os dados obtidos foram tabulados e relacionados ao peso vivo das aves, apresentados em porcentagem, antes de serem analisados estatisticamente.

Para análise estatística, empregou-se o modelo:

$$
Y_{i j k}=\mu+L_{i}+B_{j}+L_{i j}+e_{i j k}
$$

em que: $\mu:=$ constante geral; $\mathrm{L}_{\mathrm{i}}=$ efeito da inclusão de lisina; $\mathrm{B}_{\mathrm{j}}=$ efeito do nível de balanço eletrolítico $(190,255 \mathrm{mEq} / \mathrm{kg})$; $\mathrm{LB}_{\mathrm{ij}}=$ efeito da interação níveis de lisina $\mathrm{i} \times$ nível $\mathrm{j}$ do balanço eletrolítico; $\mathrm{e}_{\mathrm{ijk}}=$ erro aleatório associado a cada observação $\mathrm{Y}_{\mathrm{ijk}}$.

As características de desempenho foram submetidas à análise de regressão polinomial utilizando-se o programa SAEG (UFV, 1999). Quando a equação de regressão se apresentou quadrática, realizou-se a derivação para estimar o nível ideal de lisina, ou o nível ideal de lisina foi estabelecido pelo modelo descontínuo Linear Response Plateau (LRP), conforme melhor ajuste dos dados.

\section{Resultados e Discussão}

Os níveis de lisina influenciaram $(\mathrm{P}<0,05)$ o peso final (PF), o ganho de peso (GP), o consumo de ração (CR) e a conversão alimentar (CA) (Figura 1a), que foram melhores nos níveis 1,$53 ; 1,52$; e $1,55 \%$ de lisina, respectivamente. No entanto, o modelo descontínuo Linear Response Plateu (LRP) foi o que melhor se ajustou aos dados, estimando valores de 1,$088 ; 1,089 ; 1,138 \%$ de lisina para peso final, ganho de peso e conversão alimentar, respectivamente.

$O$ peso final dos frangos aos 40 dias de idade foi afetado de forma quadrática $(\mathrm{P}<0,05)$ pelos níveis de lisina (Figura 1b) e foi melhor no nível de $1,088 \%$ de lisina $\left(\mathrm{R}^{2}=0,95\right)$, segundo o modelo Descontínuo-LRP. Conhalato et al. (1999), avaliando níveis de lisina para frangos de corte alimentados com rações convencionais, desconsiderando 
Tabela 2 - Composição das rações experimentais (ingredientes)

\begin{tabular}{|c|c|c|c|c|c|c|c|c|c|c|}
\hline & \multicolumn{10}{|c|}{ Nível de lisina digestível (\%) } \\
\hline & \multicolumn{10}{|c|}{ Composição percentual } \\
\hline & $\begin{array}{c}\text { Basal } \\
(1,062 \% \text { lis dig. })\end{array}$ & $\begin{array}{c}\text { Basal } \\
(1,062 \% \text { lis dig. })\end{array}$ & -20 & -20 & 20 & 20 & 40 & 40 & 60 & 60 \\
\hline $\mathrm{mEq} / \mathrm{kg}$ & 190 & 255 & 190 & 255 & 190 & 255 & 190 & 255 & 190 & 255 \\
\hline Milho moído & 61,73 & 62,50 & 68,97 & 68,60 & 61,76 & 61,40 & 61,80 & 60,39 & 60,27 & 59,33 \\
\hline Glutem-milho & 3,00 & - & 8,00 & 8,04 & 3,00 & - & 3,00 & 0,07 & 3,00 & 0,12 \\
\hline Óleo de soja & 3,50 & 3,50 & 0,60 & 0,72 & 3,50 & 3,90 & 3,50 & 4,20 & 4,00 & 4,55 \\
\hline Farelo de soja & 26,31 & 30,18 & 18,43 & 18,46 & 26,28 & 30,29 & 26,24 & 30,48 & 26,56 & 30,62 \\
\hline Cloreto potássio & 0,12 & - & 0,26 & - & 0,01 & - & - & - & 0,01 & - \\
\hline Sal comum & 0,28 & - & 0,10 & - & 0,28 & - & 0,27 & - & 0,28 & - \\
\hline Bicarbonato sódio & 0,12 & 0,52 & 0,39 & 0,94 & 0,12 & 0,52 & 0,12 & 0,52 & 0,12 & 0,52 \\
\hline Calcário & 0,85 & 0,83 & 0,88 & 0,87 & 0,85 & 0,83 & 0,85 & 0,83 & 0,85 & 0,83 \\
\hline Fosfato bicálcico & 1,62 & 1,61 & 1,62 & 1,62 & 1,62 & 1,61 & 1,62 & 1,61 & 1,62 & 1,61 \\
\hline DL-metionina & 0,29 & 0,32 & 0,24 & 0,24 & 0,29 & 0,32 & 0,29 & 0,32 & 0,29 & 0,32 \\
\hline L-lisina HCL & 0,31 & 0,21 & 0,22 & 0,22 & 0,58 & 0,48 & 0,85 & 0,75 & 1,12 & 1,02 \\
\hline L-arginina & 0,12 & 0,05 & - & - & 0,37 & 0,30 & 0,62 & 0,54 & 0,86 & 0,79 \\
\hline Mistura mineral $^{1}$ & 0,06 & 0,06 & 0,06 & 0,06 & 0,06 & 0,06 & 0,06 & 0,06 & 0,06 & 0,06 \\
\hline Mistura vitamínica ${ }^{2}$ & 0,10 & 0,10 & 0,10 & 0,10 & 0,10 & 0,10 & 0,10 & 0,10 & 0,10 & 0,10 \\
\hline Coxistac & 0,05 & 0,05 & 0,05 & 0,05 & 0,05 & 0,05 & 0,05 & 0,05 & 0,05 & 0,05 \\
\hline Flavomicina & 0,004 & 0,004 & 0,004 & 0,004 & 0,004 & 0,004 & 0,004 & 0,004 & 0,004 & 0,004 \\
\hline Halquinol & 0,003 & 0,003 & 0,003 & 0,003 & 0,003 & 0,003 & 0,003 & 0,003 & 0,003 & 0,003 \\
\hline B HT & 0,02 & 0,02 & 0,02 & 0,02 & 0,02 & 0,02 & 0,02 & 0,02 & 0,02 & 0,02 \\
\hline Cloreto de colina & 0,06 & 0,06 & 0,06 & 0,06 & 0,06 & 0,06 & 0,06 & 0,06 & 0,06 & 0,06 \\
\hline Inerte & 1,47 & - & - & - & 1,05 & 0,07 & 0,55 & - & 0,72 & - \\
\hline Total & 100 & 100 & 100 & 100 & 100 & 100 & 100 & 100 & 100 & 100 \\
\hline \multicolumn{11}{|c|}{ Composição nutricional calculada } \\
\hline EM (kcal/kg) & 3.100 & 3.100 & 3.100 & 3.100 & 3.100 & 3.100 & 3.100 & 3.100 & 3.100 & 3.100 \\
\hline $\mathrm{PB}(\%)$ & 19,00 & 19,00 & 19,00 & 19,00 & 19,00 & 19,00 & 19,00 & 19,00 & 19,00 & 19,00 \\
\hline Cálcio (\%) & 0,792 & 0,792 & 0,792 & 0,792 & 0,792 & 0,792 & 0,792 & 0,792 & 0,792 & 0,792 \\
\hline Fósforo disp. (\%) & 0,395 & 0,395 & 0,395 & 0,395 & 0,395 & 0,395 & 0,395 & 0,395 & 0,395 & 0,395 \\
\hline Met.+cist. digestível (\%) & 0,849 & 0,849 & 0,849 & 0,849 & 0,849 & 0,849 & 0,849 & 0,849 & 0,849 & 0,849 \\
\hline Lisina dig. (\%) & 1,062 & 1,062 & 0,850 & 0,850 & 1,274 & 1,274 & 1,487 & 1,487 & 1,699 & 1,699 \\
\hline Arginina digest $(\%)$ & 1,238 & 1,238 & 0,990 & 0,990 & 1,486 & 1,486 & 1,733 & 1,733 & 1,981 & 1,981 \\
\hline Sódio $(\%)$ & 0,191 & 0,190 & 0,191 & 0,299 & 0,191 & 0,190 & 0,188 & 0,190 & 0,191 & 0,190 \\
\hline Potássio（\%) & 0,735 & 0,745 & 0,692 & 0,651 & 0,681 & 0,744 & 0,677 & 0,744 & 0,684 & 0,744 \\
\hline Cloro $(\%)$ & 0,320 & 0,085 & 0,268 & 0,087 & 0,320 & 0,133 & 0,360 & 0,181 & 0,420 & 0,229 \\
\hline $\mathrm{N}^{\circ} \mathrm{M} . \quad(\mathrm{mEq} / \mathrm{kg})$ & 190 & 255 & 190 & 255 & 190 & 255 & 190 & 255 & 190 & 255 \\
\hline
\end{tabular}

1 Conteúdo/kg: Fe - $100 \mathrm{~g} ; \mathrm{Cu}-16 \mathrm{~g} ; \mathrm{Mn}-150 \mathrm{~g} ; \mathrm{Zn}-100 \mathrm{~g} ; \mathrm{I}-1,5 \mathrm{~g}$.

2 Conteúdo/kg: vit. A - 10.000 .000 UI; vit. D3 - 2.200.000 Ul; vit. E - 6.000 Ul; vit. B1 - 1,4 g; vit. B2 - 4,0 g; vit. B6 - 1,8 g; vit. B12 - 15.000 mcg; ácido pantotênico - 8,5 g; vit. K - 1,4 g; ácido fólico - 0,4 g; ácido nicotínico - 25,0 g; Se - 0,3 g;

$\mathrm{NM}=$ número de Mongin $=\left[(\% \mathrm{Na}+\mathrm{x} 10000) / 22,990^{*}+(\% \mathrm{~K}+\mathrm{x} 10000) / 39,102^{*}-(\% \mathrm{Cl}-\mathrm{x} 10000) / 35,453^{*}\right]$.

em que: ${ }^{*}=$ Massa atômica do $\mathrm{Na}, \mathrm{K}$ e Cl. (Mongin,1980).

$\mathrm{N}^{0}$ de Mongin $(\mathrm{NM})=\mathrm{mEqNa}++\mathrm{mEqK}+-\mathrm{mEqCl}-(\mathrm{mEq} / \mathrm{kg})$, para se obterem 190 e $255 \mathrm{mEq} / \mathrm{kg}$

a relação entre os aminoácidos, não verificaram variações significativas no peso final das aves. Entretanto, Lana et al. (2005) e Han \& Baker (1994) observaram aumento linear no peso final conforme aumentaram o nível de lisina da dieta das aves.

Os níveis de lisina da ração influenciaram de forma quadrática $(\mathrm{P}<0,05)$ o ganho de peso dos frangos (Figura 1c), que foi menor nas aves alimentadas com as rações com menor nível de lisina. Entretanto, o modelo que melhor se ajustou foi o Descontínuo - LRP, logo, os níveis de lisina acima de $1,089 \%\left(\mathrm{R}^{2}=0,97\right)$ não melhoraram o ganho de peso das aves. Lana et al. (2005), utilizando níveis crescentes de lisina observaram efeito significativo sobre o ganho de peso aos 42 dias de idade. Conhalato et al. (1999), Costa et al. (1999) e Barboza et al. (2000) relataram resultados semelhantes, nos quais o aumento dos níveis de lisina propiciou o ganho de peso das aves. Mendes et al. (1997), no entanto, não observaram influência dos níveis de lisina sobre o ganho de peso de frangos no período de 22 a 42 dias de idade mantidos a $21,1^{\circ} \mathrm{C}$.

O consumo de ração pelos frangos de corte foi influenciado de forma linear $(\mathrm{P}<0,05)$ pelos níveis de lisina digestível (Figura 1d). Com o aumento dos níveis de lisina, houve diminuição do consumo, o que poderia ser explicado pela teoria aminoacídica de que a elevação da concentração de aminoácidos no sangue induz via hipotálamo, no sistema 


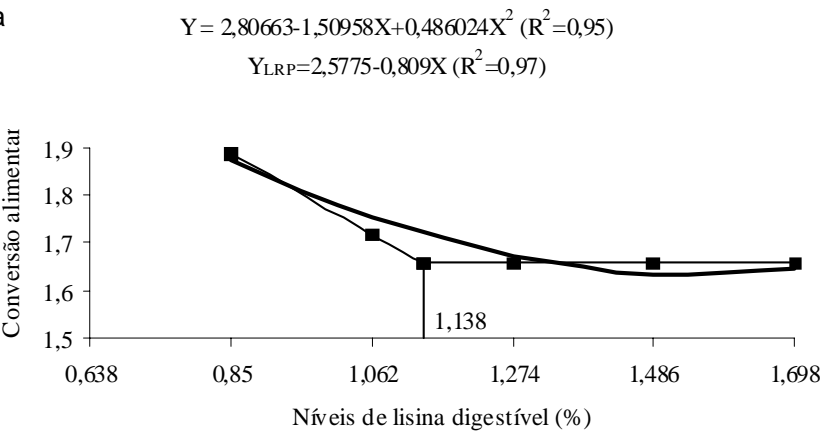

b

$\mathrm{Y}=1801,84+1125,16 \mathrm{X}-366,541 \mathrm{X}^{2}\left(\mathrm{R}^{2}=0,85\right)$

$\mathrm{Y}_{\mathrm{LRP}}=1858,4419+723,278 \mathrm{X}\left(\mathrm{R}^{2}=0,95\right)$

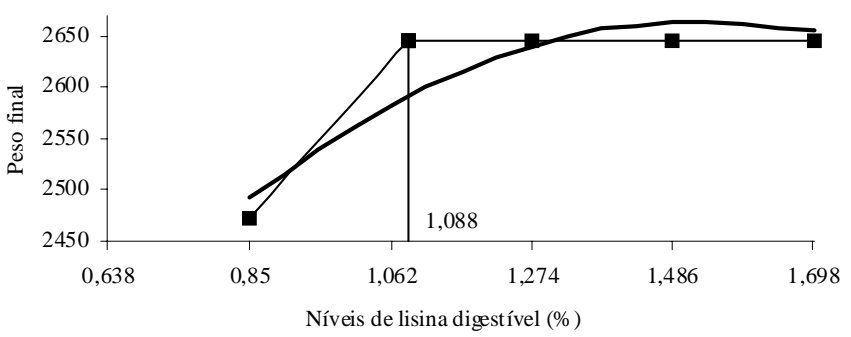

C $\quad \mathrm{Y}=889,399+1187,76 \mathrm{X}-390,511 \mathrm{X}^{2}\left(\mathrm{R}^{2}=0,87\right)$

$\mathrm{Y}_{\mathrm{LRP}}=978,3546+728,804 \mathrm{X}\left(\mathrm{R}^{2}=0,97\right)$

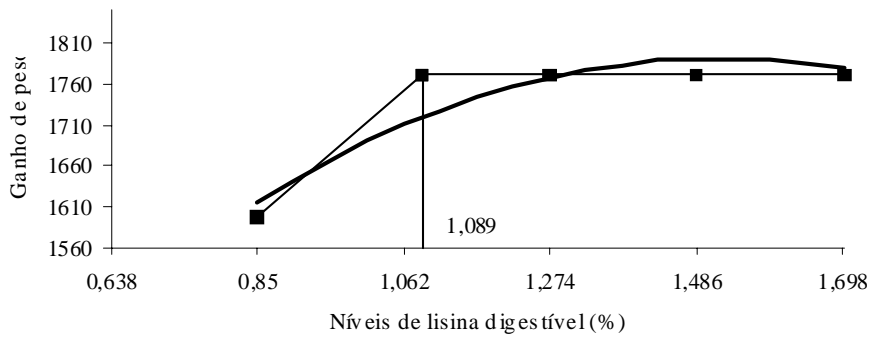

$\mathrm{Y}=3125,51-123,473 \mathrm{X}\left(\mathrm{R}^{2}=0,89\right)$

YLRP $=2,5775-0,809 \mathrm{X}\left(\mathrm{R}^{2}=0,97\right)$

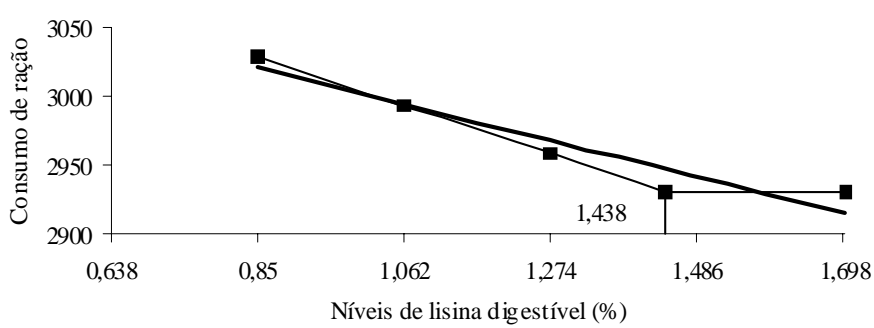

e

$\mathrm{Y}=10,3978-10,6968 \mathrm{X}+3,60771 \mathrm{X}^{2}\left(\mathrm{R}^{2}=0,43\right)$

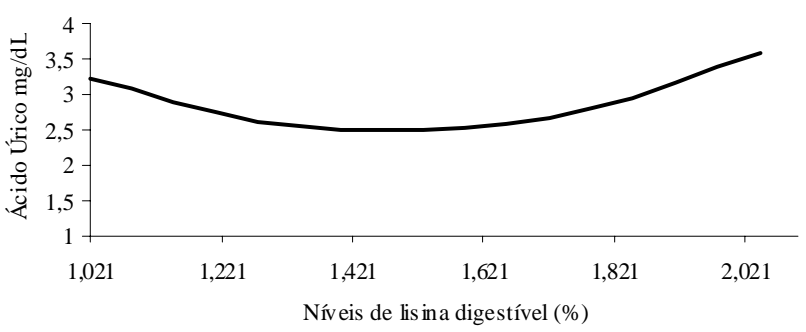

Figura 1 - Conversão alimentar (a), peso final (b), ganho de peso (c), consumo de ração (d) e concentração de ácido úrico (e) de frangos de corte de 22 a 40 dias de idade alimentados com rações contendo dois balanços eletrolíticos e diversos níveis de lisina digestível.

nervoso central, a uma redução do consumo de ração (NRC, 1987), embora não tenham sido observadas diferenças significativas no nível de proteína total no sangue das aves.

A diminuição do consumo entre as aves alimentadas com ração com altas concentrações de aminoácidos pode estar relacionada à elevação dos níveis de lisina da ração, que pode ter ocasionado competição com o potássio no mecanismo de transporte ativo da bomba sódio potássio. Neste sistema, quando o sódio é bombeado contra o seu gradiente de concentração, potássio, aminoácidos e glicose entram na célula tanto pelo transporte ativo como por difusão passiva (Nelson \& Cox, 2000). Caso entre na célula mais potássio que aminoácidos, os aminoácidos permanecerão por mais tempo na corrente sangüínea, diminuindo o consumo de alimento por inibição do centro da fome no hipotálamo. Quando há excessos de potássio, o organismo deixa de eliminar prótons para eliminar potássio, o que levaria o organismo a alcalose. A resposta fisiológica poderia ser a inibição do consumo (Granner, 1998).

Os valores de proteína total e cálcio no sangue (Tabela 4) não foram influenciados pelos níveis de lisina e pelos balanços eletrolíticos, porém o ácido úrico foi influenciado de forma quadrática $(\mathrm{P}<0,005)$ pelos níveis de lisina (Figura 1e) das dietas.

Austic \& Nesheim (1970), citados por Gadelha (2004), observaram que a maior atividade da arginase renal relacionada ao excesso de alguns aminoácidos se deve à ação de desintoxicação metabólica, que induz maior degradação de 
aminoácidos e índice de uréia nas fezes. Atualmente, no entanto, não é completo o entendimento das vias metabólicas.

Não foram encontradas diferenças significativas nos rendimentos de carcaça, peito, coxa, sobrecoxa, fígado e rim, o que confirma os resultados descritos por Han \& Baker (1994), que não verificaram melhora no rendimento de peito quando forneceram altos níveis de lisina e metionina na ração.

Almeida et al. (2002), avaliando altos níveis de lisina $(100,110$ e $120 \%)$ em rações para frangos de corte nas fases inicial, de crescimento e de abate, não encontraram dife-

Tabela 3 - Concentração de ácido úrico, proteína total e cálcio no sangue de frangos aos 40 dias de idade alimentados com rações com dois balanços eletrolíticos e diversos níveis de lisina digestível

\begin{tabular}{lccc}
\hline Nível & $\begin{array}{c}\text { Ácido úrico } \\
(\mathrm{mg} / \mathrm{dL})\end{array}$ & $\begin{array}{c}\text { Proteína total } \\
(\mathrm{mg} / \mathrm{dL})\end{array}$ & $\begin{array}{c}\text { Cálcio } \\
(\mathrm{mg} / \mathrm{dL})\end{array}$ \\
\hline \multicolumn{4}{c}{ Balanço eletrolítico $(\mathrm{mEq} / \mathrm{kg})$} \\
\hline 190 & 2,944 & 3,017 & 7,492 \\
255 & 2,958 & 3,093 & 7,237 \\
\hline \multicolumn{4}{c}{ Lisina $(\%)$} \\
\hline 0,85 & 4,234 & 3,113 & 7,691 \\
1,062 & 2,324 & 3,208 & 7,629 \\
1,274 & 3,039 & 2,850 & 6,580 \\
1,486 & 2,698 & 3,016 & 7,500 \\
1,699 & 2,455 & 3,088 & 7,422 \\
\hline \multicolumn{4}{c}{} \\
\hline Lisina & $<0,001 *$ & Fonte de variação \\
BED & - & 0,198 & - \\
BED $\times$ Lisina & - & - & - \\
CV\% & 36,739 & - & 19,765 \\
\hline
\end{tabular}

${ }^{*} \hat{Y}=10,3978-10,6968 X+3,6077 X^{2}\left(R^{2}=0,43\right)$. renças nos rendimentos de carcaça, pernas e peito, mas observaram efeito significativo sobre a deposição de gordura abdominal, que diminuiu conforme aumentou o nível de lisina. Da mesma forma, Conhalato et al. (1999) e Lana et al. (2005) não encontraram variação no rendimento de carcaça de frangos abatidos aos 42 dias de idade alimentados com ração com diversos níveis de lisina e dois balanços aminoacídicos, resultados que confirmam os relatos de que o rendimento de carcaça é pouco influenciado pelos níveis nutricionais da ração. Contudo, esses resultados diferem dos valores obtidos por Costa et al. (2001) e Vieites et al. (2005).

Não houve influência dos níveis de lisina nem dos balanços eletrolíticos sobre os pesos de rins e fígado, logo, os elevados níveis dos aminoácidos não foram tóxicos para as aves e os excessos de nitrogênio foram metabolizados normalmente pelos órgãos para formação de ácido úrico e eliminados pelo organismo.

Os níveis de lisina não influenciaram a deposição de gordura abdominal neste estudo. Costa et al. (2001), no entanto, observaram efeito linear decrescente dos níveis de lisina sobre a deposição de gordura, confirmando os resultados obtidos por Baker \& Molitoris (1991). Nesta pesquisa, a deposição de gordura abdominal foi influenciada pelo balanço eletrolítico da ração $(\mathrm{P}<0,05)$, o que pode estar relacionado ao efeito do potássio, que ativa ou funciona como co-fator de vários sistemas enzimáticos relacionados diretamente à transferência e utilização de energia e à síntese protéica (aumento da captação de aminoácidos pelas células), entre outros. Além disso, indiretamente, o potássio estimula a liberação do hormônio do

Tabela 4 - Rendimento dos cortes de frangos de corte no período de 22 a 40 dias de idade alimentados com rações com dois balanços eletrolíticos e diversos níveis de lisina digestível

\begin{tabular}{|c|c|c|c|c|c|c|c|}
\hline Nível & $\begin{array}{l}\text { Rendimento } \\
\text { carcaça }(\%)\end{array}$ & $\begin{array}{l}\text { Rendimento } \\
\text { peito }(\%)\end{array}$ & $\begin{array}{l}\text { Rendimento } \\
\text { coxa }(\%)\end{array}$ & $\begin{array}{l}\text { Rendimento } \\
\text { sobrecoxa }(\%)\end{array}$ & Fígado (\%) & $\operatorname{Rim}(\%)$ & $\begin{array}{c}\text { Gordura } \\
\text { abdominal }(\%)\end{array}$ \\
\hline \multicolumn{8}{|c|}{ Balanço eletrolítico } \\
\hline 190 & 77,814 & 24,712 & 5,231 & 10,789 & 0,00193 & 0,00026 & 0,0016 \\
\hline 255 & 77,989 & 24,776 & 5,214 & 10,664 & 0,00197 & 0,00025 & 0,0013 \\
\hline \multicolumn{8}{|c|}{ Lisina } \\
\hline 0,85 & 78,312 & 25,206 & 5,274 & 10,927 & 0,00190 & 0,00021 & 0,0015 \\
\hline 1,062 & 77,674 & 24,66 & 5,268 & 10,415 & 0,00188 & 0,00027 & 0,0014 \\
\hline 1,274 & 78,159 & 24,476 & 5,296 & 10,831 & 0,00193 & 0,00026 & 0,0015 \\
\hline 1,486 & 77,578 & 24,755 & 5,138 & 10,528 & 0,00205 & 0,00027 & 0,0016 \\
\hline 1,699 & 77,782 & 24,517 & 5,136 & 10,933 & 0,00198 & 0,00026 & 0,0014 \\
\hline \multicolumn{8}{|c|}{ Fonte de variação } \\
\hline Lisina & - & - & 0,379 & - & 0,328 & - & - \\
\hline BED & - & - & - & - & - & - & 0,037 \\
\hline BED $\times$ Lisina & - & - & - & - & - & - & - \\
\hline CV \% & 1,632 & 5,041 & 4,072 & 7,422 & 8,664 & 37,519 & 22,713 \\
\hline
\end{tabular}


crescimento $(\mathrm{GH})$, que inibe a lipogênese e aumenta a lipólise. Desta forma, os animais que receberam ração com maior balanço eletrolítico $(255 \mathrm{mEq} / \mathrm{kg})$ produziram menor quantidade de gordura abdominal, resultados que confirmam os relatos de Souza et al. (2002) de que o uso de cloreto de potássio reduz a deposição de gordura abdominal.

\section{Conclusões}

As exigências de lisina para maior ganho de peso e conversão alimentar foram estimadas em 1,089 e 1,14\%, respectivamente. Os níveis de lisina influenciaram os valores de ácido úrico plasmático, o que indica desbalanço entre os aminoácidos.

\section{Literatura Citada}

ALMEIDA, I.C.L.; MENDES, A.A.; GARCIA, R.G. et al. Efeito do nível de lisina da dieta e do sexo sobre o desempenho e rendimento de carcaça de frangos de corte. Revista Brasileira de Ciência Avícola, v.4, n.1, 8p, 2002.

BAKER, D.H.; MOLITORIS, R.A. Partioning of nutrients for growth and other metabolic functions: efficiency and priority considerations. Poultry Science, v.70, p.1797-1805, 1991.

BARBOZA, W.A.; ROSTAGNO, H.S.; ALBINO, L.F.T. et al. Níveis de lisina para frangos de corte de 22 a 40 e 42 a 48 dias de idade. Revista Brasileira de Zootecnia, v.29, p.1091-1097, 2000.

BARROS, J.M.S.; GOMES, P.C.; ALBINO, L.F. et al. Exigência de sódio para frangos de corte na fase de crescimento (22 a 42 dias) e final (43 a 56 dias). Revista Brasileira de Zootecnia, v.33, n.6, p.1721-1733, 2004.

BOORMAN, K.N. The renal reabsorption of arginine, lysine and ornithine in the young cockerel (Gallus domesticus). Comparative Biochemistry and Physiology, v.39A, p.2938, 1971 .

BRAKER, J.; BALNAVE, D.; DIBNER, J.J. Optimum dietary arginine:lysine ratio for broiler chickens is altered during heat stress in association with chabges in intestinal uptake and dietary sodium chloride. British Poultry Science, v.39, p.639-647, 1998.

CALVERT, C.C.; AUSTIC, R.E. Lysine chloride interaction in the growing chick. Poultry Science, v.60, p.1468-1472, 1981

CONHALATO, G.S.; DONZELE, J.L.; ALBINO, L.F.T. et al. níveis de lisina digestível para frangos de corte machos na fase de 22 a 42 dias de idade. Revista Brasileira de Zootecnia, v.28, p. $98-104,1999$.

CONHALATO, G.S.; DONZELE, J.L.; OLIVEIRA, R.F.M. et al. Avaliação de rações contendo diferentes níveis de lisina digestível mantendo a relação aminoacídica para pintos de corte na fase de 1 a 21 dias de idade. Revista Brasileira de Zootecnia, v.29, p.2066-2071, 2000 (supl. 1).

CORZO, A.; KIDD, M.T. Arginine needs of the chick and growing broiler. International Journal of Poultry Science, v.2, n.6, p.379-382, 2003.

COSTA, F.G.P.; ROSTAGNO, H.S.; ALBINO, L.F.T. et al. Níveis dietéticos de lisina parea frangos de corte, no período de 22 a 40 dias de idade. In: CONFERÊNCIA APINCO DE CIÊNCIA E TECNOLOGIA AVÍCOLAS, 1999, Campinas. Anais... Campinas: Fundação Apinco de Ciência e Tecnologia Avícolas, 1999. p.18.

COSTA, F.G.P.; ROSTAGNO, H.S.; TOLEDO, R.S. et al. Efeito da relação arginina:lisina sobre o desempenho de carcaça de frangos de corte de 3 a 6 semanas de idade, em condições de altas temperaturas. Revista Brasileira de Zootecnia, v.30, n.6S, p.2021-2025, 2001.

GADELHA, A.C. Resposta produtiva, imune e desenvolvimento ósseo de frangos de corte alimentados com diferentes relações de arginina e lisina digestíveis. Jaboticabal: Universidade Estadual Paulista, 2004. 171p. Tese (Doutorado em Zootecnia) - Universidade Estadual Paulista, 2004.

GRANNER, K.D. Hormônios da córtex da adernal. In: HARPER, J.C. (Ed.) Bioquímica. 8.ed. São Paulo: Atheneu, 1998. p. 707-709.

HAN, Y.; BAKER, D.H. digestible lysine requirement of male and female broiler chicks during the period three to six weeks posthatching. Poultry Science, v.73, p.1739-1745, 1994.

KIDD, M.T. Relationship between the nutritional requirements and the immune system in poultry. In: SIMPÓSIO INTERNACIONAL SOBRE EXIGENCIAS NUTRICIONAIS DE AVES E SUÍNOS, 2., 2005, Viçosa, MG. Anais...Viçosa, MG: Universidade Federal de Viçosa, 2005. p.29-31.

LANA, S.R.V.; OLIVEIRA, R.F.M.; DONZELE, P.C.G. et al. níveis de lisina digestível em rações de frangos de corte de 22 a 42 dias de idade, mantidos em ambientes de termoneutralidade. Revista Brasileira de Zootecnia, v.34, n.5, p.1624-1632, 2005.

MENDES, A.A. Efeito de fatores genéticos, nutricionais e de ambiente sobre o rendimento de carcaça de frangos de corte. Botucatu: Universidade Estadual Paulista, 1990. 103p. Tese (Livre Docência) - Universidade Estadual Paulista, 1990.

MENDES, A.A.; WATKINS, S.E.; ENGLAND, J.A. et al. Influence of dietary lysine levels and arginine:lysine ratios of performance of broiler exposed to heat or cold stress during the period of three to six weeks of age. Poultry Science, v.76, p.472-481, 1997.

MONGIN, P. Role of Sodium, potassium and chloride in eggshell quality. In: NUTRITION CONFERENCE OF FLORIDA, Florida, 1980. Proceedings... Florida: 1980. p.114-117.

NATIONAL RESEARCH COUNCIL - NRC. Nutrient requirements of poultry. 9.rev.ed. Washington, D.C.: National Academy Press, 1994. 155p.

NATIONAL RESEARCH COUNCIL - NRC. Predicting feed intake of food: producing animals. Washington, D.C.: National Academy Press, 1987. 85p.

NELSON, D.L.; COX, M.M. Biological membrane and transport. In: Lehninger principales of biochemistry. 3.ed. New York: Worth Publishers, 2000. p.389-436.

NESHEIM, M.C. Kidney arginine activity and lysine tolerance in strains of chickens selected for a high or low requirement for arginine. Journal of Nutrition, v.95, p.79-87, 1968.

ROSTAGNO, H.S.; ALBINO, L.F.T.; DONZELE, J.L. et al. Tabelas brasileiras para aves e suínos: composição química e exigências nutricionais. 2.ed. Viçosa, MG: Universidade Federal de Viçosa, 2005. 186p.

SOUZA, B.B.; BERTECHINI, A.G.; TEIXEIRA, A.S. et al. Efeitos dos cloretos de potássio e de amônia sobre o desempenho e deposição de gordura na carcaça de frangos de corte no verão. Revista Brasileira de Ciências Avícolas, v.4, n.3, p.209-218, 2002.

UNIVERSIDADE FEDERAL DE VIÇOSA - UFV. Manual de utilização do programa SAEG (sistema para análises estatísticas 8.0). Viçosa, MG: Universidade Federal de Viçosa, 1999. $141 \mathrm{p}$.

VELDKAMP, T.; SIMONS, P.C.M.; NOORDHUITZEN, J.P.T.M. et al. Effect of ambient temperature, arginine to lysine ratio and electrolytes balance on performance in commercial male turkeys. Poultry Science, v.78, p.114, 1999 (suppl.).

VIEITES, F.M.; MORAES, G.H.K.; ALBINO, L.F.T. et al. balanço eletrolítico e níveis de proteína bruta sobre o desempenho, rendimento de carcaça e a umidade da cama de frangos de corte de 1 a 42 dias de idade. Revista Brasileira de Zootecnia, v.34, n.6, p.1990-2005, 2005. 